\title{
Teaching CREdible VAlidation ANd Verification METHOdS TO A LARGE, MULTIDISCIPLINARY FirST-YEAR EngineERing Design Class
}

\author{
Vicki Komisar ${ }^{1,2}$, Andrew Flood ${ }^{3}$, Noosheen Walji ${ }^{2,4}$, Jason Foster $^{5}$ and Robert Irish $^{6}$ \\ ${ }^{1}$ Toronto Rehabilitation Institute - University Health Network; ${ }^{2}$ Institute of Biomaterials and Biomedical Engineering, \\ University of Toronto; ${ }^{3}$ Department of Electrical and Computer Engineering, University of Toronto; ${ }^{4}$ Department of \\ Mechanical and Industrial Engineering, University of Toronto; ${ }^{5}$ Division of Engineering Science, University of Toronto; \\ ${ }^{6}$ Engineering Communication Program, University of Toronto \\ V.Komisar@utoronto.ca, Andrew.Flood@mail.utoronto.ca, Noosheen.Walji@mail.utoronto.ca, \\ Jason.Foster@utoronto.ca, R.Irish@utoronto.ca
}

\begin{abstract}
This paper describes our experiences in teaching credible validation and verification methods to a class of 250 first-year Engineering Science students at the University of Toronto. While our students have previously developed proof-of-concept prototypes, this was the first year that testing their prototypes against key design requirements - and substantially integrating stakeholder feedback into their projects - were course expectations.

Core strategies to support our students included leveraging the expertise of a multidisciplinary teaching team; training students to collect and interpret data from community stakeholders; demystifying prototyping and testing through small-scale activities; and legitimizing our expectations through real-world examples.

Student design teams generally performed well with respect to validation and verification criteria on their summative project evaluations. Most teams effectively integrated stakeholder feedback with other research into developing and refining their designs, and demonstrated that their prototypes addressed key metrics. Challenges to be addressed in future course iterations are discussed.
\end{abstract}

Keywords: Validation, Verification, Engineering Design, Prototyping, Testing, Stakeholders, First-Year Initiatives

\section{INTRODUCTION}

The "Praxis" course series provides grounding in engineering design and communication for roughly 250 first-year Engineering Science students at the University of Toronto ( $\mathrm{U}$ of $\mathrm{T}$ ). In the second course in the series (Praxis II), students are tasked with "[e]ffect[ing] a verified and validated improvement in the lived experience of a community with directly-accessible representation in the Greater Toronto Area". In the context of Praxis, verification is defined as demonstrating that a design meets its key requirements, while validation involves demonstrating that a design aligns with stakeholder needs and interests.

Praxis II scaffolds this objective by having the student teams identify and frame their own design projects. The teams begin by identifying and approaching one or more communities of interest. Based on their interactions with various community and related stakeholders, each team documents a potential design opportunity in the form of a "Request for Proposals" (RFP). A core component of the RFPs is the set of requirements that any proposed solution must meet. Within the requirements, particular focus is on the metrics against which the solutions are measured.

The course teaching team selects eight RFPs from the student-developed set to proceed to the second stage of the course. These RFPs cover a diverse range of opportunities, including (most recently) finding lost arrows at Toronto's Seton Park Outdoor Archery Range; reducing the risk of pressure sores among wheelchair users with dementia at a local nursing home; and improving the process of preparing fabric orders at a local dye and fabric store. During this stage of the course, multiple teams independently design and test proposed solutions for each RFP. In keeping with the overall course objective, students are expected to both verify and validate their proposed solution during this stage.

The concepts of 'validation' and 'verification' are generally recognized as being fundamental to practicing credible engineering design (e.g. $[1,2])$. However, North American programs and institutions vary widely in when they introduce validation and verification to novice engineers, and in their depth and expectations for rigor. For example, Engineering Strategies and Practice (ESP) at $\mathrm{U}$ of $\mathrm{T}$ (a first-year engineering design and communication course sequence that includes all engineering disciplines other than Engineering Science) does not explicitly require students to prototype and test 
their design concepts. Students are expected to engage regularly with their primary stakeholder ('client'), and to obtain client approval for their preferred design concept. This model implies that verification is optional but validation, in some form, is required.

In contrast to ESP, Design Thinking \& Communication (DTC) at Northwestern University (NU) requires that its students engage in functional prototyping and user testing. DTC collaborates extensively with the Shirley Ryan AbilityLab, which provides 'clients' with disability- and rehabilitation-focused challenges. DTC students are expected to interact directly and regularly with their clients. By the end of the course, students are expected to have designed, developed, and validated proof-of-concept prototypes through direct user testing. This testing is guided by a student-designed testing protocol. In support of this expectation, students have access to a fully-equipped and staffed machine shop. This combination of technical resources and committed clients facilitates integrating validation and verification early in NU's undergraduate engineering degree programs.

Previous iterations of Praxis have required students to develop proof-of-concept prototypes and to evaluate the theoretical performance of their designs with respect to key requirements. However, the 2017 winter semester marked the first instance where verification and validation featured directly in the overall course learning objective. Meeting these objectives required students to engage in rigorous prototype testing and to solicit and integrate stakeholder feedback - simply building proof-of-concept models was no longer sufficient. This addition presented a number of challenges for a large class of first-year Engineering Science students, whose technical grounding from their other courses and life experiences were often limited. Considering the benefits and limitations of our students' diverse backgrounds and experiences, we focused on developing and supporting their skills in:

1) Identifying and prioritizing the most important ('key') design requirements for verifying and validating their designs;

2) Identifying where requirements should be modified (and then modifying them as appropriate) to enable credible testing of their design's performance in the absence of the resources that may be needed to follow 'standard' testing procedures;

3) Developing credible prototypes and testing procedures, such that students can evaluate their designs in cases that are not covered by existing codes and standards;

4) Considering the value and limitations of their prototypes and testing procedures for informing engineering design; and,

5) Collecting, interpreting, and integrating stakeholder feedback throughout their design activities.

\section{TEACHING STRATEGY}

Key elements of our strategy to teach and support validation and verification included:

1) Leveraging the skills and expertise of a multidisciplinary teaching team;

2) Training students in collecting and interpreting data from community stakeholders;

3) Demystifying prototyping and testing through smallscale, in-class activities;

4) Legitimizing validation and verification expectations through real-world examples;

5) Selecting design challenges for students that demonstrated strong community engagement and potential for solution development and verification by first-year engineering students; and

6) De-emphasizing design concept quality in favour of testing rigor during student assessments.

We expand on these teaching strategy elements below.

1. A multi-disciplinary teaching team to provide expertise in varied and unpredictable facets of engineering design. Teaching credible validation and verification techniques would not be possible without a teaching team that can offer expertise in a broad range of disciplines. In the last two years, our team has consisted of instructors and teaching assistants with backgrounds in the following engineering disciplines: aerospace, physics, electrical, systems/software, materials, nanoengineering, mechanical, chemical, infrastructure/civil, and biomedical (with wetlab, ergonomics/biomechanics, rehabilitation and clinical engineering specialties). Beyond engineering disciplines, our team includes expertise in linguistics, drama, political science, technical writing, and English literature. We also include members who are based in Toronto hospitals and collaborate with clinicians and medical device manufacturers for their graduate work.

From a verification perspective, our broad expertise provided us with the versatility to support students in developing both their prototypes and testing protocols (and any corresponding testing infrastructure, such as motion sensors) in response to a wide range of design challenges. It also helped us to calibrate what we could expect teams of first-year students to accomplish with projects of varying levels of technical difficulty.

Our teaching team's qualitative expertise was instrumental in positioning students to engage with stakeholders at all stages of their design processes, including validation. Of note, our team's expertise in qualitative research methods helped students to develop note-taking and field research strategies that enabled them to efficiently collect stakeholder feedback, and better understand how the community context could influence design requirements and uptake of their resulting designs. 
2. Field research training to scaffold skills in collecting and interpreting data from stakeholders. Many of our students had little or no experience in working with vulnerable populations, such as persons with intellectual disabilities. Similarly, many had never conducted formal field observations or collected research data from humans. Given the importance of community engagement in framing and responding to design opportunities, we thus targeted substantial course content toward enhancing student capacity in building trust with - and collecting and interpreting data from - their stakeholders.

In light of our students' inexperience in working with diverse communities, mitigating the impact of bias was vital for enabling credible verification and validation. We challenged students to consider how the language and visuals that were often used to represent various communities (e.g. villages in Tanzania) affected student preconceptions of members of these communities. We then discussed possible consequences when bias pervades a design space, using the "PlayPump" to exemplify a well-intentioned, high-visibility alternative to traditional methods of pumping water for a village. In this case, the failure of the designers to consult with community users led to a concept that was costlier than alternatives; was challenging to maintain; and did not meet recognized standards for minimum water supply [3]. Finally, we discussed strategies for reducing the impact of bias on design. We asked students to evaluate the language and visuals in their information sources about communities with whom they work, and consider how this information affects their assumptions about and interactions with the community. We also asked them to consider how their design requirements and verification/validation protocols may unintentionally reflect dubious assumptions about these groups, and to revise their protocols accordingly.

To support students in collecting data from human participants (often for the first time), we discussed strategies for building trust and facilitating conversation with community stakeholders. We leveraged content from $\mathrm{U}$ of T's Faculty of Medicine [4], including raising student awareness of how body language might influence a participant's comfort and behavior, and encouraging students to actively solicit questions from participants.

We scaffolded the process of collecting and interpreting qualitative data from potential stakeholders through a brief "Field Notes" assignment early in the semester. Students identified communities and solicited interviews and/or observation sessions to learn more about the community's context and lived experience. Students then submitted brief reports in which they summarized key findings from their observations and conversations, and discussed what these findings implied about their stakeholder's lived experience. This enabled our students to gain experience with stakeholder interactions - and learn from and receive feedback on their handling of these interactions - well before they sought to validate their designs at the end of the semester.

3. Integrating learning from other courses to demystify prototyping and help students to design credible verification protocols. While some of our students had gained substantial prototyping experience during or before their secondary education, many had minimal experience in building prototypes - let alone testing their prototypes against design requirements. As such, we wanted our students to develop basic skills not only in representing important features of their designs, but also in using those representations to evaluate their designs against key requirements, and to inform further iterations. Central components of our lessons included:

1) Highlighting that even crude and unsophisticated prototypes can be invaluable for informing design;

2) Emphasizing the importance of the question that students are trying to answer with their testing, and how that question informs both the prototype and testing protocol;

3) Introducing the notion of "pilot testing" to explicitly encourage iteration of verification protocols before testing with or on their stakeholders;

4) Introducing the concept of "proxy" metrics, to help students evaluate design performance in the absence of resources to use standard methods of verification;

5) Prompting students to consider how their prototypes and test protocol design (e.g. number of participants, number of trials per participant, presence of an inventor during subjective evaluations, accuracy and precision of measurement methods) might influence verification findings; and

6) Interrogating the distinction between "statistical" versus "functional" or "clinical" significance (i.e. whether apparent differences in the performance of various designs with respect to a given metric actually matter, and how much better a design would need to be to be considered functionally-relevant).

Where possible, we also referenced other courses where students performed experiments in subject-specific settings. This included confirmatory physics labs (where students learned about the importance of error analysis); computer programming projects (where the value of small scale unit testing becomes apparent); and failure testing in their introductory structures course (where students' theoretical calculations are scrutinized with respect to the failure test results of prototypes that they built).

To introduce our students to prototyping and testing processes within Praxis, we developed two-part tutorials in which the students prototyped solutions to improve the handwriting experience for persons with arthritis, focusing on comfort (measured via effort and finger joint angles), speed and legibility. Students were given basic 
prototyping materials (e.g. modeling clay, pipe cleaners, tape) to quickly mock up and test a variety of solutions.

- In Part 1, students re-visited design requirements in the design brief that outlined their challenge. The requirements referenced accepted measurement practices that were physically-impossible to address within their tutorial (e.g. specifying electromyography to measure the muscular activity and corresponding effort of handwriting). This prompted students to devise alternate methods of evaluating problematic requirements, such as developing subjective Likert scales to explore user perceptions of comfort.

- In Part 2, we standardized the writing utensil add-ons for students to build, including a standard pen. Students then evaluated salient finger joint angles (index finger flexion; thumb extension) that resulted from using their prototypes with respect to constraints (i.e. joint angles that could cause discomfort in persons with arthritis).

In debriefing these tutorials with the class, we focused on factors that led to the widely-divergent measurements and relative rankings of prototypes between individual teams. These factors included the students' definitions of joint angles (e.g. based on specific bones on the hand, versus defined arbitrarily), their measurement approaches, and their overall experimental design. We also discussed possible methods for simulating consequences of arthritis (in the absence of a person with arthritis to test on), such as artificially reducing a person's grip strength through cold water immersion [5], or bracing student hands with popsicle sticks to restrict range of motion. Through these short and focused activities, we aimed to help students to see that prototypes and protocols do not necessarily require sophistication to enable learning. Further, the design of their protocols can greatly impact findings, suggesting that protocols should be evaluated and refined to improve their utility for informing product design.

4. Real-world examples to legitimize training and manage expectations in validation and verification. Given the substantial effort involved with credibly validating and verifying a design concept, we sought to convince students of the importance and challenge of these processes by discussing concrete examples of where validation and verification featured heavily into design processes in industry - particularly in the early stages of these processes. We also wanted students to critically consider stakeholder feedback when evaluating their designs, rather than accepting it at face value. This concern was motivated by challenges in previous years, where students interpreted a single negative comment from a stakeholder as a complete indictment of their work - or, conversely, interpreted stakeholder comments to the effect of "your idea has potential" as "we don't need to do any more work because the stakeholder likes our design".
We leveraged products and processes to which our teaching team members contributed, and could thus discuss authentically. We focused on the rewards and challenges associated with validation and verification outside of an undergraduate course context. For example, through discussion of the first clinical installation of "StandEasy Adjustable Pole System" (a wall-mounted pole designed to help hospital patients with limited lowerlimb and torso stability to transfer out of bed more safely and independently $[6,7])$, students gained perspectives on how extremely-negative patient feedback could ensue, even when the inventors used a rigorous design process that involved ongoing feedback from clinicians, infection control specialists, maintenance staff, and end-users [8].

We also emphasized that in practice, testing with respect to design requirements and soliciting stakeholder feedback occurs throughout design processes. This differs from many traditional engineering design process models (such as the "v" model) [9], which present verification and validation as late-stage activities.

5. Careful selection and vetting of design challenges to increase the likelihood of legitimacy and stakeholder willingness to collaborate with students. In selecting student-developed RFPs for the second stage of the course, our team prioritized the verifiability of the RFP by teams of first-year Engineering Science students, as well as the apparent engagement of the host community. For example, a number of selected RFPs included letters from stakeholders (e.g. clinicians at SickKids Hospital in Toronto) who expressed both their commitment to collaborate with students for the balance of the semester and the importance of addressing the opportunity in the RFP. We also privileged design challenges that students could credibly address and verify solutions to, without requiring production-level or even functional prototypes.

\section{De-emphasizing design concept performance during assessment to encourage more rigorous verification and validation. In the past, students were mostly assessed on their design's theoretical performance with respect to key requirements; any further validation or verification was considered to exceed expectations. To reduce the risk of students downplaying or not presenting verification and validation data that did not support their designs, we emphasized the quality and credibility of their protocols (including discussion of the value and limitations of their prototypes and protocols for guiding design) in student evaluations, over the performance of the design concepts. This enabled students to achieve high grades in their assessments, even if their protocols refuted their design concepts. We aimed to avoid dis-incentivizing rigor, and instead encourage students to critically consider the extent to which their verification and validation data supported their designs as appropriate solutions to their RFPs.}




\section{STUDENT PERFORMANCE AND PROJECT HIGHLIGHTS}

The performance of the class on the validation and verification components of their culminating Design Showcase exhibition is presented in Figure 1, while the rubric descriptors for the levels shown below are presented in Appendix A. Their performance is based on project evaluations by two assessors, including at least one assessor with an engineering background and one with direct experience with this iteration of the course.

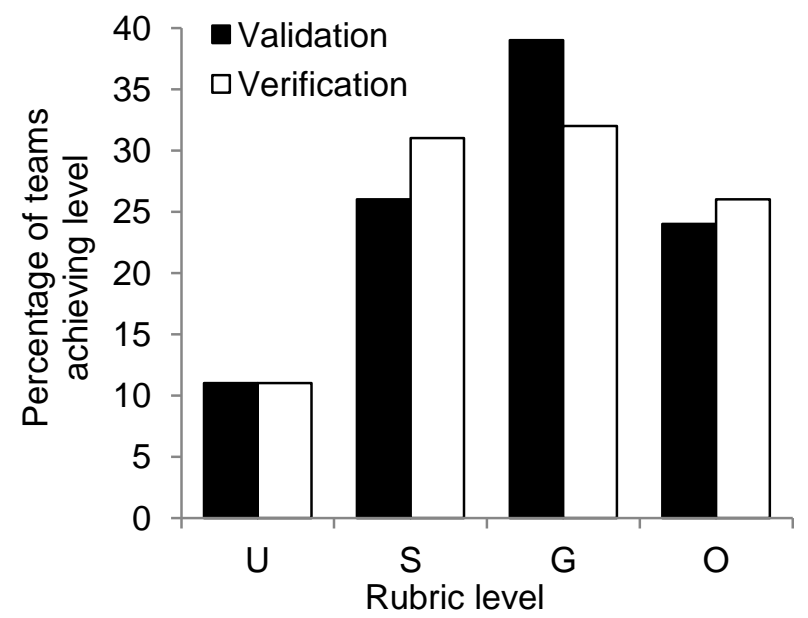

Fig. 1. Distribution of student team performance with respect to validation and verification criteria on their Design Showcase projects at the end of the semester. Values are expressed as percentages, based on a total of 50 teams. U, S, G and O correspond to rubric levels of Unacceptable, Satisfactory, Good and Outstanding.

We were encouraged by how students performed in their Showcase projects for both validation and verification. Only $11 \%$ of the class was rated 'Unacceptable' for the validation and verification criteria (i.e. stakeholder feedback was not considered in their design process; no evidence of prototype testing, calculations or modeling that address a relevant design requirement). Roughly $25 \%$ of the class was rated as 'Outstanding' for verification and validation (i.e. verification data showed that their prototype should credibly address the opportunity; stakeholder feedback suggested that their design concepts were likely to gain community acceptance).

Performances of 'Good' or higher occurred in $58 \%$ of the class for verification (i.e. credible use of verification data to inform design), and $63 \%$ of the class for validation (i.e. stakeholder feedback used to refine projects). The stronger performances in validation are likely indicative of students being limited in verification by their technical and prototyping abilities and resources. This may also reflect a more concerted effort to engage stakeholders for validation, whereas strong verification performances were often contingent on having developed testable prototypes.
Despite the challenge that verification presented to some student teams, many were able to develop protocols that were valuable for informing design. A selection of verification highlights from student projects include:

1. Some teams credibly adopted "proxy" metrics and protocols in the absence of resources or technical ability to use standard methods of verification. The teaching team was largely impressed by how many teams credibly verified their work without sophisticated equipment. For an RFP that aimed to prevent pressure sores in wheelchair users at Toronto's Kensington Gardens care home, one team mapped wheelchair seat pressure distributions with balls of clay, with greater 'squish' radii indicating higher pressure in that area. Through this, they showed that their concept reduced pressure on a person's thighs and buttocks compared to other concepts. Another team sought to better the sailing experience for sailors with disabilities, which entailed checking the effectiveness of the seat restraint. To test their solution, they used a playground swing to mimic the pitch and roll motions of a sailboat on the water, and thus gained a sense of how their seat restraint performed during wave-like motions.

2. Many teams successfully demonstrated that their intervention was an improvement over the status quo. Many students appeared to internalize our message that they had to both test their concepts, and compare their concept's performance to that of existing products. Teams aiming to facilitate the finding of lost arrows at Toronto's Seton Park archery range mostly checked their solutions against unaltered cases. For example, one solution involved changing the type of grass at the archery range, which the team tested by comparing the distance at which arrows could be sighted to that of the existing grass. In other cases, teams who modified the arrow (e.g. by including bright pink ribbons, RFID tags, or metallic tape) also tested how their alteration affected shooting repeatability by testing altered versus unaltered arrows. Many teams also thought to reduce confirmation bias by recruiting an outside expert archer to test their solutions.

3. Some teams clearly understood what constituted functionally-relevant improvements. One team aiming to reduce the risk of pressure sores in wheelchair users at the Kensington Gardens nursing home focused on temperature reduction between the user's skin and the wheelchair seat pan, which is associated with pressure sore risk. Their first round of testing demonstrated that their intervention reduced this temperature by $15^{\circ}$; their second round resulted in a less-dramatic $3^{\circ}$ reduction. However, the team was able to reference a paper that demonstrated that even a $3^{\circ}$ temperature reduction was associated with lower risk of pressure sores, and their intervention was thus still likely to be helpful. 


\section{CHALLENGES TO BE ADDRESSED}

While we were generally pleased with how these firstyear students handled validation and verification with their design projects, we experienced a number of challenges in teaching these methods that should be addressed in future iterations. Four of these challenges and potential mitigation strategies for the future - include:

1. Ensuring that admonitions to avoid inappropriately representing stakeholders do not discourage students from pilot-testing their verification protocols. We were encouraged by how students seriously considered our content on avoiding inappropriate representations of communities. However, while this likely facilitated positive community interactions, it also discouraged many teams from performing any intermediate testing between developing initial prototypes and testing on end-users, particularly for RFPs that involved communities with disabilities. We explicitly told students that they were expected to pilot-test on themselves and their peers - and thus identify and address limitations of their prototypes and protocols - before testing with stakeholders. Still, many were concerned that testing on themselves constituted inappropriate representations of their stakeholders, and thus avoided it altogether.

We were mostly successful in (eventually) convincing students to perform intermediate testing on themselves and their peers, by framing this testing as necessary due diligence. We highlighted the importance of "debugging" their protocols to ensure that their findings would be useful for guiding design, and noted that inviting others to take part in an untested protocol would be negligent. To address student discomfort, we reinforced that they were expected to acknowledge the value and limitations of their prototypes and testing protocols, and speak critically to the ecological salience of their experiments.

The general student reluctance to test their protocols before approaching end-users - despite explicit and repeated instructions to "test [their] test" - may suggest that many had not considered the possibility of their experiments failing, or felt that this risk was minimal. As such, a possible mitigation strategy could involve building experimental design into other lab courses in the first-year Engineering Science curriculum, for which their initial experimental methods may not be useful for answering their design or research questions. This may help with priming students to expect that protocol iteration will be vital in most experimental contexts, before stakeholder considerations feature heavily in their projects.

2. Maintaining sufficient and consistent community involvement for credible validation to take place. While our teaching team aimed to select design challenges that demonstrated substantial community interest and engagement, the interest (and corresponding stakeholder availability for collaboration with students) was not always consistent. In some cases, student contact with end-users (e.g. care facility residents) was limited to avoid overwhelming community stakeholders; in other cases, community contacts were enthusiastic as teams were developing RFPs, but lost interest as time passed. This was particularly problematic for communities with intellectual disabilities, whose context and experience is challenging (if not impossible) to credibly simulate for the purposes of evaluating design. Some of our teams circumvented this issue by approaching other groups, such as other care facilities with residents with dementia, or advocacy groups for individuals with disabilities.

While the precise levels of ongoing community interest can be difficult to predict, we will continue to work to select RFPs that are clearly motivated by community stakeholders - and ideally follow up with these contacts to clarify their commitment, before students attempt to solve the design opportunities within.

3. Minimizing competition between testing and alternate design activities and concepts. Whereas some students avoided testing, others over-emphasized physical experiments, at the possible expense of other methods of acquiring information, such as library research and calculations. While this may have facilitated learning among students whose strengths lie in conducting and interpreting physical experiments, it also meant that some students overlooked less expensive and potentially-more credible methods of answering questions. Another consequence was that important design considerations that were not easily tested (such as ease of manufacture) were less likely to be considered in students' work.

Given the substantial demands on students' time from other courses, we expect that this issue will be difficult to address without diluting our emphasis on verification. Introducing course content that focuses on barriers to product uptake (such as cost and manufacturing issues) framed from the perspective of key stakeholders - may help to encourage students to address less-testable features through their validation processes.

\section{Balancing the importance of discussing negative} verification and validation findings with student desires to speak positively about their design concepts. While many teams spoke cogently to the value and limitations of their designs and corresponding validation and verification processes, others were reluctant to discuss where their design concepts or knowledge fell short. After the course's final design showcase, members of our teaching team commented that some teams became defensive when asked about project weaknesses or dubious claims that they made about their stakeholders even though the student assessment criteria explicitly required discussion of where their work was limited. 
In our experience with other undergraduate design courses (including final-year Capstone courses) and even graduate contexts, many students are hesitant to refute their projects. We have not yet eliminated imbalanced discussion on project limitations. Still, we expect that by favoring critical analysis of student design processes over actual project outcomes, students may be more willing to adopt a critical perspective later in their degrees.

\section{CONCLUSION}

We have endeavored to teach credible validation and verification practices to a large class of first-year Engineering Science students with diverse cultural backgrounds, and varied technical backgrounds and interests. Core elements of our teaching strategy included leveraging the expertise of a multidisciplinary teaching team; training students in collecting and interpreting data from community stakeholders; demystifying prototyping and testing through small-scale, in-class activities; and legitimizing validation and verification expectations through real-world examples. We also aimed to select design challenges that demonstrated strong community engagement and potential for solution development and verification by first-year engineering students, and we refined student assessments to de-emphasize concept quality in favor of the rigor of their verification and validation processes.

Most student teams demonstrated credible validation and verification in their culminating Design Showcase projects, with nearly $90 \%$ of the class assessed as "Satisfactory" or higher, and roughly $60 \%$ of the class rated as "Good" or higher, with respect to both criteria. More teams were rated as "Good" or higher for validation than for verification. This may reflect the higher technical challenge of verification compared to validation.

In future iterations of Praxis, we will aim to ameliorate students' verification experiences by encouraging them to begin testing earlier, as part of vital due diligence. We will similarly aim to improve their validation potential by favoring design challenges that demonstrate strong community engagement. Toward both objectives, we will reinforce the importance of design considerations that are not easily-tested but are critical for product uptake, and remind students of their professional responsibility to speak to the strengths and limitations of their work.

We expect that first-year Engineering Science students gained a stronger understanding of the importance of developing credible validation and verification processes, and improved the overall rigor of their approach to engineering design.

\section{Conflict of Interest}

VK is an inventor of "StandEasy Adjustable Pole System", and may receive a percentage of royalties from its sale, as per Toronto Rehab-UHN's institutional policy.

\section{Acknowledgements}

The authors acknowledge funding from the Canadian Institutes of Health Research Operating Grants (CIHR MOP 142178) (VK), the AGE-WELL Network of Centres of Excellence in Technology \& Aging Graduate Student Scholarships (VK), Toronto Rehabilitation Institute Graduate Student Scholarships (VK), the Ontario Ministry for Research \& Innovation (NW), the Natural Sciences \& Engineering Research Council of Canada (NW, AF), the Training in Organ-on-a-Chip Engineering Program Scholarships (NW), and the Weber \& Mariano Graduate Scholarships (NW). We also gratefully acknowledge funding from $U$ of T's Institute of Biomaterials \& Biomedical Engineering (VK) and Division of Engineering Science (NW) to present at CEEA 2017.

\section{References}

[1] United States Food and Drug Administration, "Design Control Guidance For Medical Device Manufacturers," United States Food and Drug Administration, 11 March 1997. Available as of April 17, 2017 from https://www.fda.gov/MedicalDevices/DeviceRegulationan dGuidance/GuidanceDocuments/ucm070627.htm

[2] R. V. Welch and Z. N. Cox, "Verification and validation of Mars Exploration Rover surface capabilities," in IEEE Internation Conference on Systems, Man and Cybernetics, Waikoloa, HI, USA, 2005.

[3] R. Boland, "Radical Plumbers and PlayPumps - Objects in Development," Trinity College School of Engineering (PhD Thesis), Dublin, 2011.

[4] K. Zabjek, Disordered and Restorative Motor Control Tutorial, University of Toronto, Toronto, 2014.

[5] M. J. Vincent and M. J. Tipton, "The effects of cold immersion and hand protection on grip strength," Aviation Space and Environmental Medicine, vol. 59, no. 8, pp. 738-741, 1988.

[6] A. J. Hart, G. R. Fernie, T. D. Smyth, V. P. Komisar, E. C. King and A. M. Sobchak, "Cantilevered Support System". United States Patent 9,433,547, 6 September 2016.

[7] Hart Vision Group, "EasyRange ${ }^{\mathrm{TM}}$ 500: A wall mounted support system," Available as of May 7, 2017 from: http://www.hartvision.com/EasyRange_500Range.php. 
[8] E. C. King, V. Komisar, V. Boscart, T. D. Smyth and G. R. Fernie, "StandEasy Transfer Support System," in Toronto Rehabilitation Instittue Musculoskeletal Symposium, Toronto, ON, May 31, 2013.

[9] K. Forsberg and H. Mooz, "The relationship of system engineering to the project cycle," in INCOSE International Symposium, Chattanooga, TN, 1991.

\section{APPENDIX A: RUBRIC DESCRIPTORS FOR VALIDATION AND VERIFICATION}

Rubric levels and the corresponding descriptors for the validation and verification criteria in the course's Design Showcase are presented in Tables A1 and A2.

Table A1: Rubric levels and descriptors for the validation criterion ("The quality of the validation of the focal design concept") in the culminating Design Showcase

\begin{tabular}{|c|c|}
\hline Level & Descriptor \\
\hline 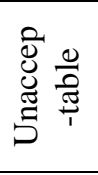 & $\begin{array}{l}\text { No evidence of stakeholder interaction or } \\
\text { collection of feedback } \\
\text { - Stakeholder feedback collected but not } \\
\text { critically considered in your design process }\end{array}$ \\
\hline 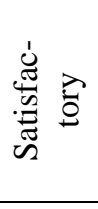 & $\begin{array}{l}\text { Stakeholder feedback collected and } \\
\text { considered in your design process, though } \\
\text { incorporation of feedback may be awkward } \\
\text { or not supported by research or other key } \\
\text { design considerations }\end{array}$ \\
\hline $\begin{array}{l}8 \\
8 \\
0\end{array}$ & $\begin{array}{l}\text { - Stakeholder feedback is integrated with other } \\
\text { research, and considered with your key } \\
\text { design requirements, in any design changes } \\
\text { or recommendations that follow from this } \\
\text { feedback }\end{array}$ \\
\hline 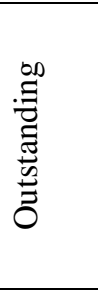 & $\begin{array}{l}\text { As per "Good" + } \\
\text { - Validation data demonstrate that your } \\
\text { concept is likely to be accepted by the } \\
\text { community } \\
\text { - Feedback from multiple, relevant } \\
\text { stakeholders is considered and incorporated } \\
\text { into your design process }\end{array}$ \\
\hline
\end{tabular}

Table A2: Rubric levels and descriptors for the verification criterion ("The quality of the verification of the focal design concept") in the culminating Design Showcase

\begin{tabular}{|c|c|}
\hline Level & Descriptor \\
\hline 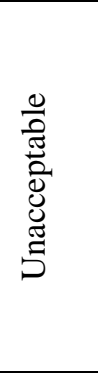 & $\begin{array}{l}\text { No evidence of prototype testing, } \\
\text { calculations, or engineering modelling } \\
\text { Verification data collected but use of results } \\
\text { shows lack of thought and due diligence } \\
\text { Verification protocol does not address a } \\
\text { metric that you can link back to the } \\
\text { opportunity and its stakeholders } \\
\text { - Strengths and limitations of test protocol are } \\
\text { not discussed }\end{array}$ \\
\hline 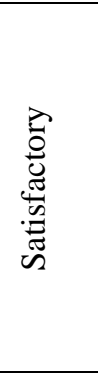 & $\begin{array}{l}\text { - Verification data collected but justification } \\
\text { for protocol or use of data may be awkward } \\
\text { - Verification protocol addresses a metric that } \\
\text { may not be the most important, but is still } \\
\text { relevant to the opportunity and its } \\
\text { stakeholders } \\
\text { - Basic strengths and limitations of protocol } \\
\text { discussed, though discussion may be } \\
\text { imbalanced or superficial }\end{array}$ \\
\hline $\begin{array}{l}\overline{8} \\
8 \\
0\end{array}$ & $\begin{array}{l}\text { Verification data used appropriately to guide } \\
\text { (or not guide) design, based on your } \\
\text { discussion of the strengths and limitations of } \\
\text { your test protocol and prototype } \\
\text { - Both the protocol itself and the metric(s) } \\
\text { that it addresses are well-justified with } \\
\text { research, logic, or other strong evidence }\end{array}$ \\
\hline 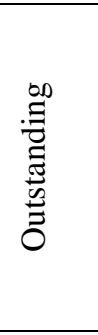 & $\begin{array}{l}\text { As per "Good" + } \\
\text { - Verification data demonstrate that your } \\
\text { prototype should credibly address the } \\
\text { opportunity } \\
\text { - Protocol credibly augments 'standard' or } \\
\text { more commonly-used procedures with your } \\
\text { own modifications, based on your context } \\
\text { and prototype }\end{array}$ \\
\hline
\end{tabular}

